\title{
Los efectos de estado de la política pública ambiental en territorios indígenas
}

\author{
Valeria Biffi Isla \\ https://orcid.org/0000-0003-3039-084X \\ London School of Economics \\ vbiffi@pucp.edu.pe
}

RESUMEN

A partir de la implementación de un programa de conservación de bosques en comunidades nativas, analizo cómo población indígena entiende al Estado como institución rectora ambiental y cómo se desempeña como usuaria de política pública. Los resultados de este estudio etnográfico sugieren que la implementación del esquema de incentivos económicos para la conservación genera una débil conexión estatal como institución ambiental, pero refuerza su papel como proveedor de oportunidades de desarrollo. Con este programa, la población indigena ha construido interpretaciones alternativas a la conservación de bosques como un activo para atraer futuras oportunidades de desarrollo y de los incentivos económicos como recompensas y propinas. Finalmente, estas desviaciones sobre la conciencia ambiental del programa se entienden por el fuerte componente administrativo sobre la rendición de cuentas que las comunidades hacen al Estado. De hecho, el principal efecto de estado del programa es la burocratización de las comunidades, con el fin de que puedan adaptarse al ideal estatal de instalar la cultura de la auditoría.

Palabras clave: efectos de estado, burocracia, pueblos indigenas, política ambiental, Amazonía. 


\section{The state effects of enviromental policy in indigenous territories}

\section{ABSTRACT}

Based on the implementation of a forest conservation program in indigenous communities analyze how the indigenous population understands the state as an environmental ruling institution and how they perform as users of public policy. The results of this ethnographic study suggest that the implementation of this scheme of economic incentives for conservation generates a weak connection of the state as an environmental institution but reinforces the role of the state as a provider of development opportunities. With this scheme, the indigenous population has constructed alternative interpretations of forest conservation as an asset to attract future development opportunities and of economic incentives as rewards and tips. The main state effect of the program is the bureaucratisation of communities to adapt then to the state ideal of installing an audit culture.

Keywords: state effects, bureaucracy, indigenous peoples, environmental policy, Amazon. 


\section{INTRODUCCIÓN}

En este artículo analizo los efectos de estado que produce la implementación del Programa Nacional de Conservación de Bosques para la Mitigación del Cambio Climático (PNCB) en población indígena kichwa y awajún en el departamento de San Martín. El PNCB es una propuesta pionera que se maneja como un esquema de incentivos económicos para la conservación de casi 3 millones de hectáreas de bosques amazónicos en más de doscientas comunidades nativas (MINAM/PNCB, 2020). El uso de los incentivos económicos está condicionado únicamente a la conservación de áreas de bosques dentro de sus comunidades, la implementación de proyectos locales de desarrollo sostenible y la conducción de patrullajes forestales en los bosques bajo acuerdos de conservación (MINAM/ PNCB, 2018, 2010). Este programa ofrece una nueva estructura de gobernanza ambiental entre el Estado y las comunidades nativas. El hecho de que las comunidades manejen sus presupuestos y organicen sus proyectos podría fortalecer el sentido de reconocimiento social y confianza de los pueblos indígenas hacia el Estado, así como reconfigurar su relación política (von Hedemann \& Osorne, 2016; Shapiro-Garza, 2013).

En este escenario, en este artículo discuto, por un lado, cómo los indígenas entienden al Estado como una institución ambiental, y por el otro, cómo se desempeñan como usuarios de política pública ambiental. Para desarrollar estas preguntas uso el enfoque de efectos de estado con el fin de enfatizar la micropolítica de las intervenciones estatales, a partir de las interacciones entre funcionarios públicos y pobladores de las comunidades nativas usuarias del PNCB. Los efectos de estado son entendidos como los rastros del control estatal en la vida cotidiana de los ciudadanos (Trouillot, 2001; Mitchell 1991). Este enfoque entiende al Estado como el resultado de prácticas implementadas a través de políticas públicas más que como una institución sólida (Mitchell, 1991). Así, los efectos de estado se ubican más bien en la performance de funcionarios públicos, en el aparato burocrático estatal, la infraestructura pública y en objetos como documentos escritos 
o tarjetas de identidad (Hull, 2012; Corbridge et al., 2005). Los efectos de estado también se determinan por las características históricas, sociales y geográficas de los contextos locales y por la intensidad, periodicidad y uniformidad de las operaciones de política pública (Hull, 2012; Painter, 2006; Corbridge et al., 2005; Gupta, 1995). El enfoque de efectos de estado al entender la construcción del Estado a partir de interacciones, objetos e infraestructura revela también el carácter efímero y elusivo de «lo estatal» (Harvey, 2005; Mitchell, 1991).

Los efectos de estado se diferencian de la «intención de estado», en la medida en que los primeros refieren al impacto o la «huella» que dejan las prácticas y procedimientos de la intervención pública — sea intencionada o no- en la sociedad, para que los individuos conduzcan ciertos comportamientos esperados siguiendo las normas propuestas. La intención refiere precisamente a la proyección de estos comportamientos y resultados esperados, que serían producto del diseño e implementación de programas de gestión pública como el PNCB. De otro lado, el imaginario estatal es el conjunto de ideas y formas de representación que tienen las comunidades y personas (Migdal, 2001). De hecho, los efectos de estado también dependen de la imagen estatal para consolidarse en la sociedad.

En el caso del PNCB, las interacciones, los documentos y objetos que se producen y circulan en la cotidianeidad del PNCB son los elementos que consolidan los efectos de estado y que hacen aparecer al Estado como una entidad material y presente en las comunidades. La idoneidad de aplicar el enfoque de efectos de estado en este caso se basa en su utilidad para comprender el Estado a través de prácticas diarias territorializadas, más que como un aparato coherente que opera de arriba hacia abajo. También permite enfocarse en la relación que se construye entre el Estado y los pueblos indígenas y examinar aquellas acciones que impactan en la forma como la población indígena experimenta el Estado y sus reglas ambientales.

Analizar los efectos de estado en comunidades de San Martín resulta importante, porque de un lado, se trata de un programa para conservar bosques en un departamento con una tasa de deforestación acumulada de aproximadamente $20 \%$ de su territorio (FAO, 2015; UICN, 2012). Esto en parte es resultado de la historia de la ejecución de una serie de proyectos de desarrollo emprendidos desde la década de 1970 que promovieron el cambio de uso de suelo para activar la economía rural de la región San Martín. Algunos de los más importantes han sido la construcción de la carretera Fernando Belaunde Terry y los «proyectos especiales de desarrollo», como el Proyecto Especial Alto Mayo. Desde la década de 2000, la política ambiental subnacional de San Martín se posicionó como 
pionera en la implementación de instrumentos de gestión ambiental con procesos de ordenamiento territorial, como la zonificación económica ecológica y luego la zonificación forestal. Algunos de estos instrumentos han afectado a la población indígena, sobre todo en sus procesos de titulación de comunidades, por ejemplo, cuando sus territorios se superponen con áreas para fines de conservación. Como explicaré más adelante, son estos procesos históricos los que han contribuido a delinear la actual relación entre el Estado y los pueblos indígenas en San Martín y que también impactan en la forma como se desempeñan como usuarios del PNCB.

De hecho, la relación entre el Estado y los pueblos indígenas en San Martín tiende a ser tensa, especialmente en las comunidades kichwa, que aún esperan la titulación de sus territorios, y entre las comunidades awajún, que tienen conflictos territoriales con invasores que el Estado se muestra reacio a resolver. Según entrevistados durante mi trabajo de campo, en San Martín el Estado no tiene la voluntad de reconocer derechos territoriales de la población indígena, por su agenda política de fortalecer la protección de la naturaleza otorgando concesiones de conservación a actores no indígenas o creando áreas de conservación regionales (Che Piu y García 2011).

\section{El estudio de caso}

El PNCB es un programa del Ministerio del Ambiente, de veinte años de duración (2010-2030), que tiene la finalidad de conservar 54 millones de hectáreas de bosques en comunidades nativas. La participación en el PNCB es voluntaria para comunidades nativas tituladas que cuentan con extensas áreas boscosas, que son de relativo fácil acceso y han tenido cierta experiencia previa con proyectos de desarrollo. Las comunidades que se afilian reciben incentivos económicos (10 soles por hectárea de bosque conservado al año por un lapso de cinco años, condicionadas no solo a la conservación de bosques sino también a la implementación de pequeños proyectos de desarrollo sostenible y al patrullaje forestal de los bosques bajo contratos de conservación. En el marco del PNCB, las comunidades son las responsables de la administración de los incentivos económicos y del manejo de sus proyectos.

En la cotidianeidad del programa, las responsabilidades administrativas demandan mucho tiempo y energía para la junta directiva, autoridad política máxima dentro de las comunidades que, como tal, opera como contraparte formal del PNCB. En San Martín, hasta fines de 2019, el PNCB tenía doce comunidades inscritas activamente, una comunidad graduada y una suspendida por mal uso de 
los incentivos económicos. En total, en San Martín, hasta el PNCB, tiene alrededor de 97000 hectáreas (PNCB, 2020). Las comunidades kichwa de Lamas y de Sisa se enrolaron en el PNCB a partir de 2016; las comunidades awajún del Alto Mayo, luego de algunos intentos fallidos del PNCB para afiliar estas comunidades desde 2012, aceptaron participar a partir de 2017.

E1 PNCB fue diseñado como parte del esquema REDD, como una iniciativa de pagos por servicios ambientales que proveen los bosques amazónicos para mitigar los efectos del cambio climático. De hecho, el programa fue lanzado en el marco de la Conferencia de las Partes de la Convención Marco de Naciones Unidas sobre Cambio Climático (CMNUCC), en 2014. Desde 1995, la CMNUCC celebra una Conferencia de las Partes (COP) anual, donde los estados y otros actores relevantes comparten sus avances para reducir las emisiones de gases de efecto invernadero. En la COP 14, en Poznan 2008, el Estado peruano anunció la creación de una estrategia de conservación para proteger 54 millones de hectáreas de bosques tropicales con el fin de mitigar los efectos del cambio climático. En la COP 15, en Copenhague 2009, el ministro del Ambiente de Perú se comprometió a reducir a cero la tasa neta de deforestación de los bosques tropicales para 2021. En 2010, el Ministerio del Ambiente creó el PNCB (MINAM, 2016).

\section{Metodología y trabajo de campo}

Para este estudio conduje una investigación etnográfica de formato inductivo en siete comunidades kichwa y awajún afiliadas al PNCB. El trabajo de campo tuvo una duración de veintidós semanas, entre 2017 y 2019. Las comunidades nativas en las que trabajé están agrupadas en tres paisajes divididos geográfica y étnicamente: dos comunidades kichwa forman parte del paisaje de Lamas, ubicado en el distrito de San Antonio de Cumbaza, provincia de Lamas; otras dos comunidades kichwa hacen el paisaje Sisa, ubicado en el distrito de San José de Sisa, provincia de El Dorado, y cuatro comunidades awajún, el paisaje Alto Mayo, ubicado en los distritos de Awajún y Moyobamba, en las provincias de Rioja y Moyobamba, respectivamente. Para el recojo de información, conduje ochenta entrevistas semiestructuradas a representantes de las comunidades nativas, tanto de las juntas directivas como comuneros en general — hombres y mujeres-, pero con un gran sesgo masculino, ya que son los hombres quienes, por lo general, asumen cargos políticos en las comunidades. También realicé observación participante de la implementación del programa, y específicamente, de la interacción entre funcionarios del programa y comuneros en las mismas comunidades. 
La dinámica del PNCB en las comunidades parte de las visitas que realizan los especialistas, por lo menos una vez al mes, para dar seguimiento al avance de las condicionalidades referidas a la implementación de los proyectos de desarro1lo sostenible y los patrullajes forestales, pero sobre todo, la documentación que representa la ejecución física y financiera de estas actividades. Es decir, el énfasis de las interacciones entre los funcionarios del programa y la población indígena es la rendición de cuentas. Esto ocurre porque cada tres meses las comunidades deben presentar informes de sus avances, que los especialistas terminan por elaborar con los insumos que entregan la junta directiva.

En las reuniones rutinarias de supervisión de avances del PNCB en las comunidades, participan entre cuatro y ocho miembros de la junta directiva comunal, que suelen ser solo hombres. El especialista del programa verifica los recibos por los gastos relacionados con las condicionalidades. En San Martín, las comunidades decidieron implementar proyectos para mejorar la productividad de cultivos de cacao en los paisajes Lamas y Sisa, y café en Alto Mayo. El especialista, junto con la junta directiva, también revisa el estado financiero del manejo de los incentivos económicos y organiza la documentación para el reporte de rendición de cuentas que demuestre el progreso del programa en la comunidad y que signifique la evidencia de la presencia del Estado en la Amazonía rural.

\section{DISCUSIÓN}

\section{El Estado, la conservación de bosques y el desarrollo}

Las comunidades nativas usuarias del PNCB reconocen el objetivo principal del programa como una iniciativa pública para conservar sus bosques comunales. Reconocen, además, que esta intervención requiere seguir un conjunto de reglas nuevas para poder seguir recibiendo los incentivos económicos, como usar este dinero para fines específicos y brindando oportunamente reportes sobre la implementación del programa. Los comuneros también tienen diferentes niveles de comprensión del programa. Evidentemente, los entendimientos más precisos provienen de los comuneros que tienen un mayor nivel de participación en las actividades del PNCB como los miembros de la junta directiva comunal, especialmente el presidente y el tesorero, principales actores responsables de la implementación local del PNCB. Después de la junta comunal vienen los participantes de las brigadas de la patrulla forestal y de la asistencia técnica en actividades sostenibles. 
Luego, el nivel de conocimiento del PNCB empieza a disminuir entre otros miembros de la comunidad. Los comuneros ordinarios, sin relación con las condicionalidades del programa, primero reciben información primero durante los procesos de socialización y afiliación, antes de firmar los contratos; luego en las reuniones mensuales que los especialistas ofrecen para explicar el avance del programa, y también en las asambleas comunales internas cada tres meses. Entre estos comuneros, se reconoce el PNCB como una iniciativa en la que se recibe dinero para cuidar los bosques, pero sin mayor detalle.

Si bien existe entendimiento sobre qué es y para qué es el PNCB en las comunidades nativas, el programa no incide en fortalecer la autoridad ambiental del Estado que regula el uso de los bosques comunales, considerando las reglas que se han propuesto para recibir los incentivos económicos, como la no deforestación, la prohibición de cultivos ilícitos, la tala, caza y pesca ilegal. Esto en parte ocurre porque, en la cotidianidad del programa, el énfasis está en el complejo proceso que significa la rendición de cuentas para la población indígena y no en promover herramientas que de hecho incidan en frenar la deforestación. Incluso en las actividades de asistencia técnica para mejorar la productividad de los cultivos aun no queda la certeza de que estos esfuerzos del PNCB permitan evitar la expansión de la frontera agrícola.

No obstante, el esquema de protección de bosques comunales a cambio de incentivos económicos ha generado interpretaciones alternativas que van más allá de la valoración de la conservación por los bienes y servicios ecosistémicos ofrecidos por los bosques para mantener los medios de vida indígenas. Para los comuneros, los bosques en pie también son un activo (o capital) que atrae oportunidades de desarrollo para sus territorios. En ese sentido, se percibe que la posesión de grandes extensiones de bosque en buen estado es un requisito que el Estado - $\mathrm{u}$ otros agentes de desarrollo - busca para decidir con qué comunidades trabajar. En los tres paisajes estudiados, se ha constituido una racionalidad política de la conservación de los bosques como instrumento para acceder a oportunidades estatales de desarrollo social, sin menoscabar la importancia de los bosques por los bienes y servicios que estos ofrecen. La noción de desarrollo que la población estudiada espera del Estado supone el bienestar colectivo, vinculado principalmente con infraestructura, carreteras, agua y saneamiento, educación y salud. De hecho, a las comunidades de PNCB les gustaría invertir los incentivos económicos de PNCB en este tipo de mejoras, en lugar de las condicionalidades que tienen que cumplir. 
Dicha definición de desarrollo como un beneficio colectivo reafirma la consideración de los indígenas amazónicos del desarrollo como una práctica relacional con el Estado. En esta relación, la materialidad del Estado como proveedor de desarrollo en las comunidades es proyectada con la presencia de infraestructura; es decir, como la vía por la cual se conectan con el Estado (Green, 2014). En cambio, la conservación de los bosques no aporta materialidad ni visibilidad estatal en los territorios indígenas, sobre todo para el caso de las comunidades kichwa de Lamas y Sisa, en las que la amenaza de deforestación ya era mínima. El reconocimiento de la relación entre los indígenas y el Estado permite la provisión de apoyo y el acceso a los recursos también subraya la identificación de las diferencias y las relaciones arraigadas de poder con el Estado. Cuando los indígenas amazónicos aceptan las reglas, condicionalidades y pagos del PNCB, también reconocen las obligaciones exigidas por el Estado - aunque, como veremos más adelante, no dejan de ser contestadas (Auyero, 2012; Hetherington, 2011). La implementación del PNCB genera una débil conexión del Estado como institución ambiental en las comunidades nativas; no obstante, el Estado no es irrelevante para la población indígena. Con la participación de las comunidades nativas en el PNCB, se refuerza la concientización sobre la importante presencia estatal para presentar sus necesidades de desarrollo. Se trata de construir una relación de intercambio con el Estado con el fin de generar beneficios para su territorio comunal (Goncalves, 2016).

\section{La población indígena como usuaria de política pública ambiental}

Si bien la población indígena de comunidades usuarias del PNCB tiene un entendimiento relativamente claro de los objetivos del PNCB, las razones atribuidas a la provisión de incentivos económicos generan diferentes interpretaciones, lo que afecta la forma en que pobladores de dichas comunidades nativas se desenvuelven como usuarios de política pública. Estas interpretaciones revelan aspectos interesantes sobre la relación que los pueblos indígenas amazónicos intentan configurar con el Estado.

\section{Los kichwa}

De acuerdo con los resultados del trabajo de campo, para los pobladores kichwa de Lamas y Sisa el PNCB significa que, por primera vez, son recompensados económicamente por el Estado por su labor histórica de conservación de bosques. Sin embargo, también entienden que esta recompensa no es gratuita. Ello implica 
que deben corresponder al Estado con el cumplimiento de las condicionalidades formales del PNCB: desde participar en las reuniones mensuales a las que son convocados hasta elaborar informes de avances del programa y rendición de cuentas. En estas reuniones, por lo general, los comuneros que participan son miembros de la junta directiva y de otros comités asociados con las actividades sostenibles que se fomentan como parte del PNCB. En estas reuniones, los participantes se aburren y no necesariamente entienden lo que se está exponiendo, pero saben que es su obligación estar presentes como beneficiarios del apoyo estatal. Para garantizar la presencia del mayor número de comuneros en estas reuniones, la junta comunal impone una multa económica a los que faltan.

Con la reinterpretación de los incentivos económicos, las comunidades kichwa construyen su relación con el Estado, situándose en la posición moral de reciprocidad. La necesidad de «devolver» o reciprocar con el Estado no está considerada en términos monetarios, sino a través del cumplimiento cívico de condicionalidades administrativas y económicas, con su participación responsable en actividades y eventos propuestos, así como con el de las reglas de la PNCB. A cambio - y a razón de su conducta-, las comunidades kichwa esperan que el Estado pueda incrementar su presencia en su territorio con oportunidades de desarrollo. Esta reinterpretación local del PNCB en términos de reciprocidad recuerda la clásica definición del don, definida por Mauss (1954). Para Mauss, las transacciones aparentemente económicas para intercambiar bienes y servicios entre individuos o grupos en realidad se basan en la economía del don, que implica dar, recibir y devolver.

En el caso de las comunidades kichwa, la reflexión instrumental en el PNCB tiene la finalidad de construir una relación social, moral y política con el Estado a través de un mecanismo de intercambio mutuo. El cumplimiento de las condicionalidades se trata, entonces, de reconectar con el Estado, expandiendo su legitimidad dentro de sus comunidades y reafirmando su interés en valorar y esperar su presencia activa con servicios y asistencia en desarrollo. Pero, para hacerlo, deben comportarse siguiendo las reglas, de lo contrario no obtendrán los beneficios estatales. De hecho, las comunidades kichwa - sobre todo las de Lamas - no tienen mayores inconvenientes en la implementación del PNCB. Las reuniones convocadas por el PNCB son las que tienen la más alta participación de comuneros.

En este escenario, los kichwa de Lamas y Sisa se sitúan como usuarios pasivos del Estado que esperan su presencia, la cual debe ser reciprocada (Auyero, 2013). Así, para los kichwa, las intervenciones estatales están justificadas porque 
la «ayuda» que reciben no es vista como un derecho. De hecho, las comunidades kichwa de Lamas son consideradas por autoridades públicas locales y organizaciones sin fines de lucro como usuarios cumplidos de proyectos de desarrollo. En San Martín, las comunidades kichwa de Lamas suelen ser las candidatas preferidas para implementar proyectos, debido a su entusiasmo y responsabilidad para participar en estas iniciativas.

Los comuneros kichwa, como sujetos de beneficio, acogen la mayoría de los proyectos públicos de desarrollo que llegan a sus comunidades con la expectativa de mejorar sus medios de vida y de construir una relación activa y dinámica con el Estado. Este sentido de ganarse al Estado puede que no se sume a la reciprocidad ambiental que se requiere en el esquema del PNCB, pero crea un sentido de inclusión política y social y de reconocimiento de la presencia estatal en los márgenes.

En la historia reciente de San Martín, la condición de la población kichwa como «sujetos de beneficios» (Correa y Roopnaraine, 2014), como enfoque que condiciona su relación con el Estado, también se fundamenta en la consolidación tardía del movimiento indígena kichwa en la región para reclamar derechos diferenciados, como sus derechos territoriales y también servicios públicos básicos. Antes de los 1990, los kichwa en San Martín optaban por minimizar los rasgos característicos de su indigeneidad para evitar la discriminación que las élites mestizas locales ejercían sobre ellos, sobre todo en cuanto a la comercialización de productos agrícolas y del bosque. Esta élite, además, ha colocado históricamente a las principales autoridades políticas sin mayor preocupación por insertar de manera equitativa a la población indígena en la sociedad mayor. De ese modo, muchas familias preferían establecerse en asentamientos rurales, como el resto de población rural mestiza, en lugar de hacerlo en comunidades nativas.

Fue recién a partir de la experiencia de otros grupos indígenas en la región amazónica, y del apoyo de organizaciones sin fines de lucro, que desde finales de la década de 1980, los kichwa comenzaron a construir su movimiento político local con miras a exigir al Estado el reconocimiento de sus derechos territoriales como indígenas y la constitución de comunidades nativas. La Federación de Comunidades Kichwas de la Región San Martín (FEPIKRESAM) se estableció en 1987. Los fundadores de FEPIKRESAM fueron profesores bilingües de Lamas que, luego de participar en un evento de educación bilingüe organizado por AIDESEP y la Universidad Nacional de la Amazonía Peruana (UNAP), decidieron crear una organización política para representar a los kichwa en San Martín (Mendoza, 2013). 
Sin embargo, hasta el día de hoy, la población kichwa de San Martín no ha logrado culminar sus procesos de titulación, sobre todo por superposición con otros derechos de uso e incompatibilidades con el proceso de zonificación territorial de la región (Chaparro y Valderrama, 2017). El largo historial de dependencia de la población kichwa con referencia la sociedad mestiza también ha incidido en la construcción de un ideal de desarrollo proveniente de una fuente externa, como el Estado, y asumiendo una relación de subordinación de la que esperan protección y ayuda y, a cambio, ofrecen cumplir con su rol cívico de usuarios de intervenciones públicas (Mendoza, 2013; Calderón, 2003, 2000).

\section{Los awajún}

Las comunidades awajún en Alto Mayo definen los incentivos económicos del PNCB como favores y propinas estatales y consideran que estos pagos no necesitan ser reciprocados. Con los incentivos reinterpretados de este modo, las autoridades awajún tienen menos estímulos para obedecer las reglas del PNCB. De hecho, Alto Mayo es el paisaje con más dificultades para cumplir con las condicionalidades del PNCB. Por ejemplo, las reuniones entre los especialistas del programa y miembros de las juntas directivas se cancelan de vez en cuando porque las autoridades locales no llegan, llegan tarde y en alguna ocasión alguien llegó borracho. Los pobladores awajún también se resisten a realizar patrullajes forestales, aludiendo que estas actividades tendrían que ser pagadas por el programa, ya que los comuneros dejan de hacer otras actividades económicas por cumplir con esta condicionalidad.

En estas comunidades, también es difícil lograr que las juntas directivas compilen sus comprobantes de pago de los gastos por el PNCB para la rendición de cuentas. En una ocasión, durante una asamblea comunal, el presidente de la comunidad manifestó haber comprado equipos (impresora, cámara de fotos y otros) por un total de cinco mil soles, pero no tenía ningún comprobante para corroborar la compra y tampoco pudo enseñar los equipos que había comprado. A diferencia de las comunidades kichwa, las comunidades awajún no tienen este sentido de la importancia moral del cumplimiento de condicionalidades en la medida en que no les afecta fracasar como usuarios de política pública. De hecho, durante trabajo de campo, alguna de estas comunidades fue alertada sobre posibles pérdidas de contratos por no cumplir con la documentación de rendición de cuentas.

Estas percepciones de la población awajún sobre los incentivos económicos del PNCB como propinas y favores refleja una forma más distante de relacionarse 
con el Estado. Los comuneros awajún, especialmente los líderes, entienden al Estado como una entidad precaria, con capacidad desigual de brindar servicios públicos en los márgenes estatales, como las comunidades nativas. Para los awajún, el Estado debe ofrecer servicios públicos y promover iniciativas de desarrollo local porque es su responsabilidad. A diferencia de las comunidades kichwa, los awajún proponen tener una relación no recíproca con el Estado, pues tratan los pagos del PNCB como un derecho debido a la histórica ausencia estatal con políticas en favor de los pueblos indígenas. En este escenario, los awajún actúan políticamente como sujetos de derechos exigiendo la presencia estatal en sus comunidades, aludiendo a su vulnerabilidad como poblaciones relegadas. Esta delicada relación que se construye entre el Estado y las comunidades awajún del Alto Mayo se ve reforzada por las autoridades estatales locales, que expresamente prefieren no trabajar con las comunidades awajún del Alto Mayo por considerarlas problemáticas, refiriéndose a su supuesta falta de voluntad para seguir las reglas en los proyectos estatales y por ser «vagos», con poca motivación para participar (comunicación personal con gerentes de desarrollo social y económico de la Municipalidad de Rioja).

La condición de «sujetos de derechos» de los awajún del Alto Mayo, que también condiciona la relación con el Estado peruano, debe entenderse considerando experiencias pasadas y fallidas de intervenciones estatales para modernizar el valle del Alto Mayo. Importantes esquemas desde la década de 1970, como la construcción de la carretera Fernando Belaunde y el Proyecto Especial Alto Mayo contribuyeron a definir la tensa relación que estas comunidades tienen con el Estado en la actualidad. Desde este tiempo, y en nombre de la modernización, los pueblos awajún han sido desposeídos de sus territorios tradicionales y han sido víctimas de iniciativas fracasadas de desarrollo agrícola que los convirtieron en deudores de préstamos agrarios (Greene, 2009). Hoy, los awajún de Alto Mayo discuten y evalúan aceptar o rechazar proyectos, según la conveniencia y esfuerzo que deben como usuarios de las iniciativas. En ese sentido, los awajún están dispuestos a construir una relación frágil con el Estado, a romperla y luego a reanudarla, si fuese necesario. Cabe recordar aquí que las comunidades awajún del Alto Mayo son las que en más ocasiones han rechazado la presencia del PNCB.

En la historia reciente de San Martín, a diferencia de los kichwa, la creación del movimiento indígena awajún se empezó a consolidar desde la década de 1970 para incidir en procesos de titulación de comunidades en el Ato Mayo, detener la presencia de invasores y servir de intermediarios con agentes de proyectos para modernizar el valle a través de proyectos de desarrollo agrícola (Brown, 2014; 
Greene, 2009). En ese contexto, se creó la Organización Aguaruna del Alto Mayo (OAAM) como organización de representación política. En 2004, la OAAM se convirtió en la Federación Regional Indígena del Alto Mayo (FERIAAM), que está activa hasta el día de hoy.

En cuanto a las formas locales de interpretar los incentivos económicos, las comunidades de ambos grupos étnicos tienen diferentes interpretaciones que producen diferentes formas de relacionarse con el Estado. En lo que coinciden es que los incentivos económicos del PNCB se interpretan fuera de preocupaciones ambientales. Los kichwa se refieren a los incentivos económicos como recompensas estatales que deben ser retribuidas moralmente en forma de cumplimiento diligente de las reglas del PNCB. Por el contrario, para los awajún, los incentivos económicos son favores o propinas del Estado y, por tanto, no necesitan ser devueltos ni obligan a cumplir con las reglas del PNCB.

Estas formas diferentes de abordar los pagos revelan las formas contrastantes que estos grupos indígenas amazónicos se relacionan con el Estado. Estos entendimientos impactan en la forma en que actúan como usuarios de política pública como fuente de desarrollo. Los kichwa se enmarcan como sujetos de beneficio, tratando de mantener una relación constante y dinámica con el Estado. Los awajún se posicionan como sujetos de derechos, cuestionando la autoridad estatal, pero aun tratando de construir una relación de trabajo con él, incluso si eso significa a veces romper relaciones.

La presencia estatal en estas comunidades es suficiente para potenciar el reconocimiento político de los usuarios indígenas amazónicos como interlocutores estatales y para aceptar las iniciativas de desarrollo como una práctica relacional para fortalecer la relación Estado-pueblos indígenas.

La suspicacia de los incentivos económicos en la relación entre Estado y comunidades nativas

Pese a la aceptación de los incentivos económicos y condicionalidades del PNCB en comunidades nativas, este programa genera formas sutiles de desconfianza y sospecha hacia el Estado, que se pueden considerar con lo que Scott (2008) define como discursos ocultos o infrapolítica. La infrapolítica se refiere a las estrategias, discursos y acciones de resistencia de grupos oprimidos que permanecen invisibles para sus dominadores. En el caso del PNCB, la infrapolítica refiere a las formas locales de objeción sobre las normas y condicionalidades del programa, particularmente con respecto al desacuerdo sobre el valor económico 
de sus bosques comunales y a la discrepancia por no poder invertir los incentivos económicos en usos alternativos fuera de lo establecido, pero que según las comunidades contribuyen con el desarrollo local, tales como caminos, agua y saneamiento, local comunal, etcétera.

Las comunidades cuestionan el monto de los incentivos económicos pagos que reciben. Estas argumentan que el valor de sus bosques en pie es superior a los 10 soles por hectárea que reciben. Los especialistas normalmente no explican la cantidad total de dinero que estas recibirán por el total de la duración de sus contratos, por cinco años, sino solo el precio de 10 soles por hectárea de bosque conservado, lo cual, en términos reales, es el precio de dos o tres cervezas. Visto de ese modo, la cantidad de dinero que reciben las comunidades por proteger los bosques y cumplir con las condicionalidades del PNCB parece insignificante y las comunidades sienten una desatención estatal. Si se compara esta tarifa — que es plana para todas las comunidades - con el precio de mercado de sus tierras, especialmente, entre comunidades awajún del Alto Mayo que arriendan sus tierras para agricultura, los incentivos económicos parecen más insignificantes aún. En el valle del Alto Mayo, el alquiler de una hectárea para fines agrícolas puede llegar a costar hasta mil soles. Pese a las narrativas de rechazo al valor de los incentivos económicos, las comunidades igual aceptan los pagos, no solo porque, como se ha mencionado, es «mejor que no recibir nada», sino porque, además, las transacciones del PNCB, a través de las interacciones con especialistas, flujos de información y documentos y transacciones económicas, les permiten reconectarse con el Estado.

Además de la disconformidad por los pagos del PNCB, las comunidades también sospechan sobre otros posibles intereses estatales al brindarles dinero a cambio de conservación. De vez en cuando aparecen narrativas y temores sobre posibilidades de despojo y pérdida del territorio comunal indígena por los pagos que reciben. Entre estas narrativas, he podido escuchar argumentos sobre los incentivos económicos como pagos disfrazados de empresas extranjeras para extraer recursos naturales, o compensaciones para en un futuro crear áreas naturales protegidas. De hecho, estos discursos hacen eco con preocupaciones habituales de la población indígena sobre la seguridad legal de sus territorios. En San Martín, una de las principales razones que obstaculizan la titulación de comunidades nativas es la superposición con otros derechos de uso, como áreas de conservación, concesiones forestales, bosques de producción permanente y también con las propuestas de zonificación territorial. 
A la fecha, los desacuerdos sobre la forma de conducción del PNCB no ha escalado hacia las autoridades nacionales de manera formal; más bien, se mantienen en el nivel de quejas informales en la cotidianeidad de las interacciones con los especialistas del programa. Para los usuarios de PNCB, hacer públicas están demandas podría ponerlos en riesgo de no recibir ningún pago del todo. Así, la infrapolítica del PNCB no se trata de romper la relación de los pueblos indígenas con el Estado, sino de un intento por encontrar mejores formas de ajustar la regulación estatal a las necesidades y expectativas de los indígenas amazónicos (Rubenstein, 2004).

En términos generales, la implementación del PNCB muestra el efecto complejo y contradictorio sobre la presencia estatal en las comunidades nativas. Por un lado, las comunidades han aceptado voluntariamente participar del programa y cumplen sus condicionalidades (sobre todo las kichwa), y por el otro, las comunidades ejecutan estas actividades con cierto sentido de resignación sobre los incentivos económicos que reciben y, aunque presente de manera latente, con cierto temor sobre un potencial despojo de sus tierras. Entonces, con el PNCB, más que un compromiso con la conservación de los bosques, lo que está en juego es la posibilidad de llevar el Estado a los márgenes.

\section{La burocratización de comunidades como efecto de estado}

Un efecto importante de la implementación del PNCB es la burocratización de las comunidades. Esto se refiere a los intentos estatales, a través del programa, de instalar y reproducir formas burocráticas estatales en comunidades nativas para organizar y ejecutar acciones públicas y gestionar su relación con la población indígena a partir de la producción y circulación de documentos y trámites administrativos. La burocratización de las comunidades supone el ejercicio de inculcar habilidades administrativas y el lenguaje burocrático estatal, para que los indígenas puedan desempeñarse como usuarios activos y legibles de la política pública. De hecho, la burocratización de las comunidades empezó con las primeras afiliaciones en San Martín, desde 2016, con la exigencia de instalar un comité de gestión que respondiera por las condicionalidades y responsabilidades administrativas del programa, así como el manejo de los incentivos económicos. Se trataba de una forma de organización paralela a la autoridad política local representada por la junta directiva. No obstante, rápidamente, la tensión entre ambas organizaciones por el control del programa empezó a generar conflictos internos y motivó al programa a dejar sin efecto esta forma de organización 
y proponer que las responsabilidades administrativas recaerían sobre la junta directiva como máxima autoridad política en las comunidades.

De ese modo, las autoridades locales en las comunidades nativas deben demostrar su capacidad para administrar los incentivos económicos y rendirle cuentas al Estado, a través de informes en hojas de cálculo, que muestran gastos y saldos correctos, además de presentar otros documentos de gestión, como declaraciones juradas de gastos, reportes de actividades, entre otros. En otras palabras, las comunidades deben demostrar que forman parte de la cultura de la auditoría que propone el Estado y que están dispuestos a participar de esta estrategia de control estatal, que aquí también deja ver que poco tiene que ver con la conservación de bosques. Así, a partir de la burocratización de las comunidades, desde la plataforma de un programa ambiental, se engendran nuevas normas de conducta, leguajes y habilidades.

Para el Estado, la burocratización de las comunidades es la vía para obtener la evidencia más efectiva de su relación social con la población indígena y del avance de sus intervenciones en los márgenes estatales, sellada en trámites y documentos legales (Bear y Mathur, 2015). Por su parte, las comunidades han asumido y valoran estos canales de intercambio con el Estado. De hecho, en sociedades indígenas amazónicas, la alfabetización —y especialmente la escritura— han sido, y aún son, formas importantes de mediación para acceder al mundo occidental e interactuar con agentes externos, especialmente aquellos que se espera que contribuyan a mejorar sus comunidades, como el Estado (Gonzales, 2016; Walker, 2016). Los pueblos indígenas entienden que la escritura y la documentación los colocan en una situación más ventajosa para negociar con el Estado, la sociedad en general y el mercado (Allard y Walker, 2016; Espinosa, 2016).

La conexión con los trámites burocráticos estatales no es un escenario nuevo instalado por la PNCB. En realidad, se extiende más allá de la implementación del programa a otras agencias estatales. Por ejemplo, desde mediados de la década de 1970, los pueblos indígenas amazónicos conocieron la importancia de las actas, certificados, documentos de identificación e informes para obtener títulos legales sobre sus territorios ancestrales (Greene, 2009). Hoy, los trámites burocráticos son acciones cotidianas en la vida de los pueblos indígenas amazónicos. Las autoridades locales de los miembros de la junta comunal viajan constantemente a las ciudades cercanas para cumplir con los procedimientos burocráticos estatales relacionados con los asuntos comunales, como actualizar tarjetas de identidad, legalizar documentos, presentar solicitudes para mejorar su escuela local, completar formularios para actualizar los estatutos comunales, solicitar la mejora de una carretera, etcétera. 
En las comunidades, la relación con la burocracia estatal ya son parte de la vida cotidiana. En trabajo de campo, mientras realizaba observación participante de una reunión entre una especialista del PNCB y mujeres artesanas en una comunidad kichwa de Lamas, de pronto un hombre interrumpió la reunión $\mathrm{y}$, sin saludar, pidió copias de los documentos de identidad de las mujeres. En ese momento, las mujeres reunidas fueron rápidamente a sus casas y trajeron las copias de sus DNI, sin mayor cuestionamiento. Al reanudar la reunión del PNCB, comentaron que de vez en cuando reciben visitas de funcionarios de agencias estatales locales, que les piden sus documentos de identidad para incorporarlos en propuestas de proyectos de desarrollo. Por eso, siempre tienen guardadas varias copias de sus DNI. También mencionaron, que, en la mayoría de las ocasiones, estos proyectos no se ejecutan.

Para las comunidades nativas, los documentos y procedimientos administrativos del PNCB, como elaborar documentos de rendición de cuentas, llenar y firmar cheques, escribir declaraciones juradas, presentar boletas, escribir reportes de patrullajes, entre otros, son acciones clave para generar habilidades que les permiten aprender a redactar «solicitudes» y acceder al Estado, destrezas que además refuerzan las expectativas colectivas de acceso a las oportunidades de desarrollo (Walker, 2016). De hecho, las comunidades del PNCB anhelan acceder a esquemas de desarrollo, sin intermediarios burocráticos, como podrían ser federaciones indígenas locales y regionales, funcionarios públicos y representantes de ONG. Y para hacerlo, necesitan dominar —e incluso imitar — los procedimientos burocráticos estatales en sus propias estructuras de gobierno. Así, aceptan la burocratización de sus comunidades como condición necesaria para conectarse con el Estado y el mundo de la asistencia para el desarrollo.

El rasgo característico del PNCB que incide en la burocratización de las comunidades es que estas son las responsables de administrar el dinero que el Estado les ofrece por conservar sus bosques. Ello conlleva una serie de trámites y procedimientos burocráticos que van desde la firma de un contrato con el Estado hasta la rendición de cuentas sobre el uso de los incentivos económicos. El efecto de la burocratización de hecho se mantiene constante con las interacciones cotidianas con los especialistas, quienes realizan sus «rituales de verificación» de que, en efecto, las comunidades ejecutan lo que el Estado les exige (Power, 1997).

Con esta carga administrativa, sin proponerlo formalmente, el PNCB espera que las autoridades locales se conviertan en burócratas comunales que posean ciertas habilidades para rendir cuentas, considerando dicha capacidad como un valor público fundamental en la reconstrucción de la relación del Estado con 
los pueblos indígenas. De hecho, la mayoría de los problemas en el avance del PNCB en estas comunidades están vinculados con errores administrativos más que con fallas en evitar la deforestación, como, por ejemplo, por documentos no enviados o gastos no rendidos. Sin embargo, en esta relación, los indígenas del PNCB reconocen el poder de los documentos para validar su relación con el Estado y asumen su subordinación a los procedimientos estatales.

Durante trabajo de campo, en una reunión con los especialistas del PNCB en la que había que firmar cheques, me sorprendió las dificultades que enfrentaban los miembros de la junta directiva y de algunos comités de actividades económicas para llenarlos. Algunos de ellos no podían encajar palabras y números sobre los espacios establecidos en los cheques: lo hacían o muy arriba o muy abajo. Se tomaban su tiempo practicando la escritura de cantidades de dinero, sobre todo montos en miles de soles. También practicaban sus firmas para que les saliera igual en los documentos que deberían enviar al programa.

El PNCB, en representación estatal, subestima la comprensión de la escritura como una capacidad neutral y transparente para acceder al Estado, sin considerarla como una práctica cultural, cargada de valor simbólico, incrustada en relaciones de dominación textual (Messick, 1992). Esto sobre todo en población indígena que suelen estar por debajo del promedio en indicadores de calidad de vida y acceso a servicios públicos, incluida la educación. Entre la población indígena, el promedio del nivel educativo alcanzado es primaria incompleta. Así, el Estado, a través del PNCB, espera que las comunidades respondan al proceso de burocratización como usuarios de las políticas, pero no está dispuesto a contribuir en la construcción de estas habilidades.

La dificultad para producir documentos no solo se desprende de la falta de habilidades administrativas, sino también a la ilegibilidad de los procedimientos estatales. Aquí, la ilegibilidad es consecuencia de la incapacidad estatal para traducir sus normas, documentos legales y trámites burocráticos en forma comprensible para las comunidades nativas. Esta ilegibilidad no se fundamenta necesariamente en el desconocimiento de la regulación por los indígenas, sino en la incapacidad estatal para traducir sus normas en la vida cotidiana de los márgenes y sus dinámicas sociales y territoriales.

En este escenario, algunas comunidades optan por estrategias «informales» para cumplir con las fechas de entrega de la documentación de rendición de cuentas, aunque esto signifique sacrificar la transparencia de los procesos administrativos. En alguna ocasión, en trabajo de campo he sido testigo de reclamos de comuneros sobre pedidos de la junta directiva de firmar por su asistencia en 
actas de asambleas comunales que no se llevaron a cabo. Cuando los especialistas del PNCB confrontaron a los miembros de la junta por hacer firmar eventos fantasmas, estos afirmaron que no tenían otra opción, porque la fecha de la asamblea comunal no encajaba con el plazo que tenían para entregar su documentación y eso podía poner en riesgo el contrato. En otra reunión de rendición de cuentas, los miembros de la junta presentaron declaraciones juradas incorrectas y tuvieron que escribirlas nuevamente. Pero los comuneros que debían firmar las declaraciones no estaban en la comunidad en ese momento. Los comuneros que sí estaban en la reunión falsificaron firmas y las huellas dactilares, como si fueran las de sus vecinos ausentes.

Estos ejemplos demuestran que la cotidianidad y mayor demanda de energía de este programa ambiental está en la reproducción de prácticas burocráticas abrumadoras para la población indígena. Además, demuestran que esta intervención estatal está diseñada para adaptarse a la cultura de auditoría estatal y para reproducir sus procedimientos burocráticos, pero no a la dinámica y estructura de las comunidades nativas en cuestión como usuarios de las políticas públicas. De modo que el PNCB reproduce la ilegibilidad que significa el aparato burocrático estatal para las comunidades, lo que impacta en el alejamiento de los usuarios del PNCB del propósito mismo del programa, pero que los acerca al lenguaje de la burocracia, entendida además como una técnica de desarrollo. A pesar de esta complejidad, las comunidades están dispuestas a seguir tratando de lidiar con la burocracia y la documentación estatal. Cuando las comunidades envían sus informes de rendición de cuentas, esperan que sus contratos legales sigan siendo válidos, pero en un sentido más simbólico, también esperan que sus contratos sociales con el Estado se mantengan con la expectativa de acceder a más beneficios y oportunidades de desarrollo.

En este artículo discuto que la implementación del PNCB en comunidades nativas de San Martín fortalece la comprensión del Estado como fuente de desarrollo más que como una institución reguladora del uso del territorio indígena. Sin embargo, el PNCB crea una interpretación alternativa para la conservación de los bosques. La población indígena usuaria del PNCB reconoce la importancia de mantener los bosques en pie como un activo colectivo que atrae la presencia de fuentes externas de desarrollo, sobre todo el Estado. Este programa promueve la construcción de un perfil de las comunidades para ser receptoras de política pública para el desarrollo que incluya dominar la alfabetización burocrática y la conservación de extensas áreas boscosas. 
En cuanto a los incentivos económicos del $\mathrm{PNCB}$, las comunidades interpretan estos pagos de manera diferente, pero coinciden en que están al margen de las preocupaciones ambientales. Para los kichwa, los incentivos económicos son recompensas que deben ser reciprocadas al Estado en términos morales, con su buen comportamiento como usuarios de política pública. Para los awajún, los incentivos son favores o propinas estatales y, como tales, no merecen reciprocidad. Así, estos últimos están más dispuestos a crear, quebrar y retomar la relación con el Estado, porque consideran que es su obligación. En cambio, los kichwa prefieren mantener una relación constante y dinámica con el Estado.

La poca visibilidad de las preocupaciones ambientales en la cotidianeidad del PNCB en las comunidades depende del énfasis a los procedimientos burocráticos para que las comunidades operen como usuarios legibles de política pública, sobre todo para rendir cuentas por el manejo de los incentivos económicos. En este escenario, la población indígena ha aceptado la burocratización de sus comunidades como una estrategia de acercar el Estado a sus territorios. De hecho, la burocratización de las comunidades nativas a partir de la implementación del PNCB también representa la expansión estatal en los márgenes, legitimando el control de sobre comportamiento ambiental dentro de sus territorios y obligando a sus estructuras políticas a reproducir métodos administrativos basados en tecnologías de legibilidad política (Allard y Walker, 2016).

Así, el Estado interactúa y negocia la implementación de esta iniciativa de conservación en términos de documentación legible y una cultura de auditoría, pero pierde la oportunidad de trabajar con las comunidades en temas realmente afines al PNCB, como evitar la deforestación en sus territorios. Sin embargo, más allá de sus objetivos ambientales, el PNCB revela una nueva forma compleja y tensa de participación en la relación entre el Estado y la población indígena: una que opera entre aceptación y resistencia, legibilidad e ilegibilidad. El PNCB es una plataforma para que las comunidades se articulen políticamente con el Estado para el acceso a los servicios públicos y la creación de futuras oportunidades de desarrollo. Al mismo tiempo, la participación de las comunidades en el PNCB indica un involucramiento instrumental que les obliga a apalancar sus habilidades burocráticas y aumentar su agencia para presentar su legibilidad política al Estado.

El principal efecto de estado es su representación como una fuerza externa ausente, compuesta de una serie de procedimientos burocráticos y reglas a las cuales ceñirse si es que se espera acceder a sus recursos. De hecho, queda claro que para la población indígena la burocracia es una técnica de desarrollo. En el caso del PNCB, los indígenas encontraron que la mejor manera de lidiar con la 
barrera burocrática es aprender sobre ella, su idioma y formas. Entonces, a pesar de que el Estado ha fallado consistentemente a los pueblos indígenas en la construcción de políticas públicas sólidas, de alguna manera insisten en mantener sus símbolos e incorporar las formas estatales como a través de la burocratización de sus comunidades (ver también De Vries, 2010). Estos resultados demuestran el interés de las poblaciones indígenas en reconocer al Estado como autoridad y como una entidad que les puede otorgar medios para ejercer su ciudadanía.

Así, uno de los efectos más importantes que resulta pertinente para las comunidades es justamente la necesidad de hacerse legible ante el Estado. Con ello, los comuneros del PNCB en San Martín se esfuerzan por compartir el lenguaje de la gobernanza estatal para ser usuarios legibles de la política pública, quienes en la cotidianidad están regulados por el Estado a través de la rendición de cuentas más que en el uso de su territorio. Así, el control en el que quiere incurrir el Estado basado en la administración del gasto y de la ejecución física se distancia de actividades para conservar bosques en el departamento de San Martín, que tiene el porcentaje de deforestación acumulada más alto del país y donde la presión sobre los bosques para ampliar la frontera agrícola, va en aumento.

\section{Agradecimientos}

Esta investigación fue financiada gracias a FONDECYT-CONCYTEC (Contrato $\mathrm{N}^{\circ}$ 230-2015-FONDECYT).

\section{REFERENCIAS BIBLIOGRÁFICAS}

Allard, Oliver y Walker, Harry (2016). Paper, power, and procedure: Reflections on Amazonian appropriations of bureaucracy and documents. The Journal of Latin American and Caribbean Anthropology, 21(3), 402-413. https://doi.org/10.1111/ jlca. 12237

Auyero, Javier (2011). Patients of the state: An Ethnographic Account of Poor People's Waiting. Latin American Research Review, 46(1), 5-29.

Brown, Michael (2014). Upriver: the turbulent life and times of an Amazonian people. Cambridge: Harvard University Press. https://doi.org/10.4159/ harvard.9780674735583

Calderón, Luis (2000). Los kichua lamistas: una experiencia de campo. Arqueología y Sociedad, 13, 199-208. Museo de Arqueología y Antropología de la Universidad Nacional Mayor de San Marcos. 
Calderón, Luis (2003). Relaciones interétnicas entre nativos y mestizos en Lamas en el contexto de la globalización. En C. Degregori (ed.), Comunidades locales y transnacionales. Cinco estudios de caso en el Perú (pp. 13-104). Lima: IEP.

Chaparro, Anahí y Valderrama, Miguel (2017). Políticas de conservação e direitos territoriais dos povos indígenas: O caso da região San Martín, Perú. Habitus, 15(1), 73-92. https://doi.org/10.18224/hab.v15i1.5901

Che Piu, Hugo y García, Tania (2011). Una aproximación a los servicios ambientales a través de las propuestas de Ley Forestal y de Fauna Silvestre y, Ley de Provisión de Servicios Ambientales. Boletín REDD, edición No 4. Lima: DAR.

Corbridge, Stuart; Williams, Glyn; Srivastava, Manoj y Véron, René (2005). Seeing the State: Governance and Governmentality in India. Cambridge: Cambridge University Press.

De Vries, Pieter (2010). Redimir la promesa utópica del desarrollo: hacia un mundo, una mundialización, una modernidad. En V. Bretón (ed.), Saturno devora a sus hijos. Miradas criticas sobre el desarrollo y sus promesas (pp. 87-126). Barcelona: Icaria.

Espinosa, Oscar (2016). La política indígena en la ciudad: el caso de las comunidades urbanas Shipibo-Konibo. En Alexandre Surrallés, Oscar Espinosa y David Jabin (eds.), Apus, caciques y presidentes: Estado y política indígena amazónica en los países andinos (pp.97-116).

FAO (2015). Los bosques y el cambio climático en el Perú. Roma: FAO.

Gonçalves, Johanna (2016). Opening a Path with Papers: Yanomami Health Agents and Their Use of Medical Documents. In Allard, Oliver and Walker, Harry (Eds). Paper, power, and procedure: Reflections on Amazonian appropriations of bureaucracy and documents. The Journal of Latin American and Caribbean Anthropology, 21(3), 434-456. https://doi.org/10.1111/jlca.12237

Greene, Shane (2009). Customizing Indigeneity: Paths to a visionary politics in Peru. Palo Alto: Stanford University Press.

Gupta, Akhil (1995). Blurred boundaries: the discourse of corruption, the culture of politics, and the imagined state. American Ethnologist, 22(2). Oxford: Wiley.

Harvey, Penelope (2005). The Materiality of State-Effects: An Ethnography of a Road in the Peruvian Andes. En Christian Krohn-Hansen y Knut Nustad (eds), State Formation: Anthropological Perspectives (pp. 123-141). Londres: Pluto Press.

Hetherington, Kregg (2011). Guerrilla auditors: the politics of transparency in neoliberal Paraguay. Durham N.C.: Duke University Press.

Hull, Matthew (2012). Documents and bureaucracy. Annual Review of Anthropology, 41(1), 251-267. https://doi.org/10.1146/annurev.anthro.012809.104953 
Mauss, Marcel (1954). The gift: forms and functions of exchange in archaic societies. Londres: Routledge \& Kegan Paul.

Mendoza, Rafael (2014). Forests with history: Exploring the social effects of the creation of the Cordillera Azul National Park on the chazutino people of Amazonian Perú. Tesis para obtener el grado de doctor en Antropología por la Universidad de Florida.

Messick, Brinkley (1992). The Calligraphic State: Textual Domination and History in a Muslim Society. Berkeley: University of California Press.

Migdal, Joel. (2001). State in society: studying how states and societies transform and constitute one another. Cambridge University Press.

Ministerio del Ambiente (2016a). La conservación de bosques en el Perú. Conservando los bosques en un contexto de cambio climático como aporte al crecimiento verde. Lima: MINAM.

Ministerio del Ambiente (2016b). Estrategia de Bosques y Cambio Climático. MINAM.

Ministerio del Ambiente/Programa Nacional de Conservación de Bosques (2010). Creación del Programa Nacional de Conservación de Bosques para la Mitigación del Cambio Climático. MINAM.

Ministerio del Ambiente/Programa Nacional de Conservación de Bosques (2018). Conservación de bosques comunitarios. A través de las transferencias directas condicionadas. Balance 2010-2017. MINAM.

Ministerio del Ambiente/Programa Nacional de Conservación de Bosques (2020). Memoria Institucional del Programa Nacional de Conservación de Bosques para la Mitigación del Cambio Climático al 2019. MINAM.

Mitchell, Timothy (1991). The limits of the state: Beyond statist approaches and their critics. The American Political Science Review, 85(1), 77-96.

Painter, Joe (2006). Prosaic geographies of stateness. Political Geography, 2006-09, 25(7), 752-774. https://doi.org/10.1016/j.polgeo.2006.07.004

Power, Michael (1997). The audit society: $R$ rituals of verification. Oxford: Oxford University Press.

Rosa da Conceição, Hugo; Börner, Jan y Wunder, Sven (2015). Why were upscaled incentive programs for forest conservation adopted? Comparing policy choices in Brazil, Ecuador, and Peru. Ecosystem Services, 16, 243-252 https://doi. org/10.1016/j.ecoser.2015.10.004

Scott, James (2008). Domination and the arts of resistance: hidden transcripts. New Haven: Yale University Press. 
Shapiro-Garza, Elizabeth (2013). Contesting the market-based nature of Mexico's national payments for ecosystem services programs: Four sites of articulation and hybridization. Geoforum, 46, 5-15. https://doi.org/10.1016/j. geoforum.2012.11.018

Surrallés, Alexandre; Espinosa, Oscar; Jabin, D. (2016). Introducción. En A. Surrallés, O. Espinoza, D. Jabin (eds.), ¿Apus, caciques y presidentes? Estado y política indígena amazónica en países andinos. Lima: IWGIA.

Trouillot, Michel (2001). The Anthropology of the state in the age of globalization. Current Anthropology, 42(1), 125-138.

UICN (2016). Una mirada integral a los bosques de Perú. Quito: UICN.

vonHedemann, Nicoletta y Osorne, Tracey. (2016). State forestry incentives and community stewardship: A political ecology of payments and compensation for ecosystem services in Guatemala's highlands. Journal of Latin American Geography, 15(1), 83-110. https://doi.org/10.1353/lag.2016.0002

Walker, Harry (2016). Documents as Displaced Voice: Writing among Amazonian Urarina: Writing among Amazonian Urarina. The Journal of Latin American and Caribbean Anthropology, 21(3), 414-433. https://doi.org/10.1111/jlca.12244

Works, Marianne (1984). El proceso de desarrollo y la experiencia de cambio: situación de los aguarunas del Valle del Alto Mayo. Amazonía Peruana, 5(10), 119-128. 